Emerging therapy review

\title{
FTY720 in multiple sclerosis: the emerging evidence of its therapeutic value
}

\author{
Andrew Thomson
}

Core Medical Publishing, Knutsford, UK

\begin{abstract}
Introduction: Multiple sclerosis is a demyelinating disease of the central nervous system which can cause severe disability and has profound effects on patients' quality of life over several decades. Although there is no cure for the disease, recently developed diseasemodifying agents have modest effects on the impact of disease progression. There is therefore a need for a new, effective, and welltolerated treatment for multiple sclerosis and FTY720 (an orally administered immunomodulatory compound with a novel mechanism of action) is one of a number of agents being evaluated for the treatment of this disease.
\end{abstract}

Aims: The objective of this article is to assess the therapeutic potential for FTY720, now in phase II clinical trials, for the treatment of multiple sclerosis through a review of the published evidence.

Emerging evidence: There is good evidence that FTY720 achieves immunomodulation as shown by a reversible redistribution of peripheral blood lymphocytes after oral administration. Two meeting abstracts have been published showing results obtained with FTY720 in a 12-month phase II clinical trial in patients with active relapsing multiple sclerosis. There is modest evidence that FTY720 significantly improves both patient-oriented (relapse rate) and disease-oriented outcomes (inflammatory disease activity). There is good evidence that FTY720 is well tolerated.

Profile: Based on these early results from the clinical development program, FTY720 has the potential to be an effective diseasemodifying agent for the treatment of multiple sclerosis. Further results from ongoing multinational phase III studies are awaited.

Key words: evidence-based review, FTY720, immunomodulator, multiple sclerosis

\section{Core emerging evidence summary for FTY720 in multiple sclerosis}

\begin{tabular}{|ll|}
\hline Outcome measure & Emerging evidence \\
\hline $\begin{array}{l}\text { Patient-oriented evidence } \\
\text { Disease relapse rates }\end{array}$ & $\begin{array}{l}\text { Reduction in relapse rates and time to first relapse } \\
\text { Likelihood that patients will at least have longer intervals between relapses } \\
\text { Daily oral dosing with or without food } \\
\text { Nonvenient administration }\end{array}$ \\
$\begin{array}{ll}\text { Nell tose alterations necessary with hepatic impairment } \\
\text { transient reduction in heart rate }\end{array}$ \\
$\begin{array}{l}\text { No evidence of increased risk of infections associated with drug-related lymphocyte sequestration } \\
\text { Disease-oriented evidence }\end{array}$ \\
$\begin{array}{l}\text { Reduction of new and existing inflammatory lesions responsible for subclinical disease progression } \\
\text { magnetic resonance imaging }\end{array}$ \\
Immunomodulation & $\begin{array}{l}\text { Reversible lymphocyte sequestration, a characteristic of the mode of action of FTY720, is a convenient } \\
\text { surrogate marker of immunomodulation }\end{array}$ \\
\hline
\end{tabular}




\section{Scope, aims, and objectives}

Multiple sclerosis is one of the most common chronic neurologic diseases causing progressive disability in young adults. The life expectancy of patients with multiple sclerosis is at least 25 years following the first onset of symptoms and most patients will die from unrelated causes. In recent years there has been great progress in understanding the pathogenic mechanisms associated with the disease and imaging techniques have been developed to monitor the effects of treatment on neurologic lesions. However, although recently developed disease-modifying agents have improved the management of multiple sclerosis, there is still no treatment that stops the development of disability.

FTY720 is a novel immunomodulatory compound in clinical development for use in the prevention of organ rejection in transplant patients and for multiple sclerosis.

The objective of this review is to evaluate the evidence for the potential of FTY720 as a treatment for multiple sclerosis.

\section{Methods}

The English language medical literature was reviewed for relevant articles on FTY720 for the treatment of multiple sclerosis. An initial search of PubMed, BIOSIS, and EMBASE was conducted on June 13, 2005 using the search terms "FTY720 OR FTY720 AND multiple sclerosis" for articles published between January 1993 and June 2005 (inclusive). In addition, relevant abstracts were identified from the annual scientific sessions of the European Neurological Society, the American Society for Neurochemistry, and the International Society of Neuroimmunology, held during 2002 and 2005. The online database, www.clinicaltrials.gov was searched for information on ongoing phase II and phase III studies with FTY720 in multiple sclerosis. A hand search of reference lists in selected publications was carried out to ensure that no relevant articles were omitted.

A total of 16 articles (14 full papers and two abstracts) was identified from the initial search strategy after any animal, in-vitro, or other nonrelevant publications were omitted (Table 1). All of the full papers identified initially were excluded from the evidence evaluation. Only one meeting abstract was included for analysis as it reported pertinent clinical outcomes with FTY720. Following the initial search strategy a further six full papers were identified from reference lists in the excluded full publications for inclusion in the review of evidence. The search strategy was also repeated on January 10 , 2006 when one further relevant meeting abstract was identified and included. Thus, a total of eight publications (six full papers and two meeting abstracts) were included in the evidence base.

\section{Disease overview}

\section{Signs and symptoms}

Multiple sclerosis is one of the most common neurologic diseases affecting young adults. It is usually a disease with sporadic episodes and is characterized as a variably progressive disorder

\section{Table 1 | Evidence base included in the review}

\begin{tabular}{|lcc|}
\hline Category & Number of records \\
\cline { 2 - 3 } & Full papers & Abstracts \\
\hline Initial search & 14 & 2 \\
records excluded & 14 & 1 \\
records included & 0 & 1 \\
Additional studies identified & 6 & 1 \\
Level 1 clinical evidence & 0 & 0 \\
Level 2 clinical evidence & 5 & 2 \\
Level $\geq 3$ clinical evidence & 1 & 0 \\
trials other than RCT & 0 & 0 \\
case reports & 0 & 0 \\
Economic evidence & 0 & 0 \\
Total records included & 6 & 2 \\
\hline For definition of levels of evidence, see Editorial Information on inside back cover. \\
RCT, randomized controlled trial.
\end{tabular}

of the nervous system in which patchy degenerative inflammatory changes occur within the brain and spinal cord (Compston \& Coles 2002). The symptoms of multiple sclerosis are diverse and can include tremor, paralysis, loss of bladder or bowel control, fatigue, pain, loss of cognitive function, disturbances in vision and speech, emotional changes, and nystagmus. These symptoms can have a profound effect on patients' quality of life and can also lead to significant reliance on their family, dependents, and carers.

The severity and prognosis of multiple sclerosis can vary greatly. In about a quarter of all patients the disease does not affect activities of daily living. However, severe disability can affect about $15 \%$ of patients within a relatively short period of time (Compston \& Coles 2002) and approximately half of all patients will require a cane for walking short distances within about 15 years of first onset of the disease (Weinshenker 1994). Attacks can occur randomly, with an initial incidence of about one per year followed by a steady increase in subsequent years.

\section{Epidemiology}

The incidence of multiple sclerosis is estimated to be seven cases per 100000 per annum, and the prevalence is approximately 120 cases per 100000 . The lifetime risk of the disease is one in 400 (Compston \& Coles 2002). There are about 2.5 million individuals with multiple sclerosis in the world, and in the USA alone there are about 350000 affected patients (Lutton et al. 2004). Multiple sclerosis develops in twice as many women as men and age at onset of the disease is usually $20-30$ years. About $5 \%$ of all cases occur in patients under the age of 16 years.

\section{Etiologic, genetic, and environmental factors}

The relationship between genetic and environmental factors in determining the susceptibility of patients to develop multiple sclerosis is complex and poorly understood. However, it is clear that there is an uneven geographic distribution of the disease in populations of northern European origin and an increased 
prevalence in geographically temperate areas (Dyment et al. 1997). Thus it is a disease that predominantly affects northern Europeans.

To date the major histocompatibility complex (MHC) is the only area of the human genome with a clear association with the disease. Results from three genomic searches imply that a number of genes with interacting effects will ultimately be found; however, to date no single genetic region has been identified with a major influence on familial risk (Dyment et al. 1997). In addition, attempts to implicate specific environmental agents as responsible for the disease have been unsuccessful. Possible, but as yet unsubstantiated, candidate agents include Chlamydia pneumoniae and human herpes virus 6 (Compston \& Coles 2002).

\section{Pathophysiology}

Multiple sclerosis is characterized by acute focal inflammatory demyelination and the loss of axons with limited remyelination (Noseworthy et al. 2000). This leads to the presence of characteristic multifocal sclerotic plaques in the white matter of the central nervous system. These lesions are particularly common in the optic nerves, and white matter tracts of the periventricular regions, brain stem, and spinal cord (Hafler 2004). Typically $\mathrm{T}$ and $\mathrm{B}$ lymphocytes, macrophages, and antibodies can be found at the site of white matter destruction.

A number of fundamental questions remain regarding the pathophysiology of multiple sclerosis. For example, what initiates the inflammation and what is the antigenic target driving the inflammation (Hafler 1999)? Possible triggers for the initial inflammatory insult include an autoimmune response (initiated by autoreactive $\mathrm{T}$ lymphocytes) or a structural alteration in the white matter as a result of microbial infection. It has also been hypothesized that multiple sclerosis is a spectrum of diseases and that some are initiated by an autoimmune response and others are induced by viral infections of the central nervous system (Hafler 1999). It is unlikely that the antigenic target driving the disease is due to a single antigen. The inflammatory process initiated by T-cell recognition of one myelin protein epitope subsequently leads to the activation of autoreactive $\mathrm{T}$ cells recognizing other epitopes of the same protein. This "epitope spreading" can lead to activation of $\mathrm{T}$ cells recognizing other myelin proteins that may get degraded and be presented on the MHC of local antigen-presenting cells (Hafler 1999).

It is known that trauma does not induce multiple sclerosis, nor does trauma activate a latent form of the disease or alter symptoms in a patient with the disease. However, the risk of an exacerbation in a patient with multiple sclerosis has been shown to be associated with stressful life events (Mohr et al. 2004). As yet, specific stressors cannot be linked to exacerbations and patients themselves should not be led to believe that they bear responsibility (through experiencing stress) for them.

\section{Diagnosis}

The typical stimulus for patients to seek medical help is the first acute attack. An accurate clinical history and a thorough neurologic examination are crucial for the accurate diagnosis of multiple sclerosis. At present there is no specific immunologicbased test for the disease. Results from imaging investigations should be used to support the clinical diagnosis and to rule out other pathologies. In the absence of clinical evidence, abnormalities detected by imaging are insufficient grounds for a diagnosis (Miller et al. 1998). Annual magnetic resonance imaging (MRI) scans are also recommended for the management of ongoing multiple sclerosis to monitor disease progression and to detect underlying pathology.

MRI has both prognostic and diagnostic applications in multiple sclerosis. It has a pivotal role in the diagnosis of the disease and acts as a surrogate marker of drug efficacy in clinical trials. The use of imaging technology has been important in demonstrating that even during apparently stable periods between attacks the disease is still very active (Miller et al. 1998).

\section{Classification and clinical course}

At onset, multiple sclerosis can be categorized clinically as either relapsing remitting multiple sclerosis (RRMS) or primary progressive multiple sclerosis (PPMS). The most common form of the disease is RRMS, which is observed in about $85 \%$ of all patients (Fig. 1). RRMS is characterized by clearly defined disease relapses with full recovery or with sequelae and residual deficit upon recovery. On average about 1.5 attacks occur each year and approximately 10 new lesions are detected annually on MRI scan (Hafler 1999). Although RRMS is not classified as a progressive form of multiple sclerosis, residual deficits may occur after each exacerbation. At least half of all patients with RRMS will transition to secondary progressive multiple sclerosis (SPMS). This subform is characterized by disease progression with or without occasional relapses, minor remissions, and periods of stability. In contrast, PPMS is seen in far fewer patients (about 10\%; Fig. 1).

\begin{tabular}{|c|c|c|}
\hline Classification & $\begin{array}{l}\text { Type of incidence } \\
\text { at presentation }\end{array}$ & $\begin{array}{l}\text { Schematic typical } \\
\text { of clinical course }\end{array}$ \\
\hline $\begin{array}{l}\text { Relapsing remitting } \\
\text { multiple sclerosis } \\
\text { (RRMS) }\end{array}$ & $\sim 85 \%$ & 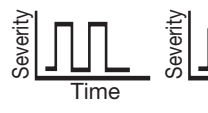 \\
\hline $\begin{array}{l}\text { Secondary progressive } \\
\text { multiple sclerosis } \\
\text { (SPMS) }\end{array}$ & $\begin{array}{l}\sim 50 \% \text { of patients } \\
\text { with RRMS will } \\
\text { progress to this } \\
\text { subform }\end{array}$ & 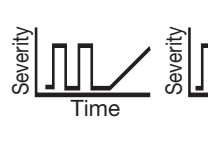 \\
\hline $\begin{array}{l}\text { Primary progressive } \\
\text { multiple sclerosis } \\
\text { (PPMS) }\end{array}$ & $\sim 10 \%$ & 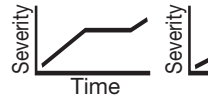 \\
\hline $\begin{array}{l}\text { Progressive relapsing } \\
\text { multiple sclerosis } \\
\text { (PRMS) }\end{array}$ & $\sim 5 \%$ & $\underbrace{\frac{3}{d}}_{\text {Tim }}$ \\
\hline
\end{tabular}

Fig. 1 | Classification, incidence, and examples of clinical courses of subtypes of multiple sclerosis (adapted with permission from Kieseier \& Hartung 2003) 
It is characterized from the outset by the absence of acute attacks but demonstrates a worsening in disease severity.

Progressive relapsing multiple sclerosis (PRMS) is the least common form of the disease, affecting about $5 \%$ of patients. From the outset it is progressive, with or without full recovery, and progression is continuous between relapse periods.

Schematic representations of the courses of these four forms of multiple sclerosis are shown in Fig. 1. During a relapse, symptoms can develop over hours to days, persist for several days or weeks, and then gradually dissipate.

\section{Prognosis}

Patients with sensory or visual symptoms as the dominant feature, particularly those who experience complete recovery from attacks, generally have the best prognosis. This pattern is common in younger women (Compston \& Coles 2002). Prognosis is particularly poor in males when disease onset occurs later in life, and in patients with frequent and prolonged relapses (particularly in the first 2 years) and in those with a short interval between the initial attack and the first relapse (Noseworthy et al. 2000) (Table 2).

\section{Current therapy options}

The aim of treatment of multiple sclerosis is to reduce the frequency (and limit the lasting effects) of relapses, relieve symptoms, prevent disability arising from disease progression or incomplete recovery from relapses, and promote tissue repair (Compston \& Coles 2002).

The management of multiple sclerosis has greatly benefited from the availability of five disease-modifying agents which have been approved by the US Food and Drug Administration (FDA) since 1993 and are now widely available. However, there is no cure for the disease and available disease-modifying agents are lifelong therapies. Other therapies may be used to alleviate some of the chronic symptoms of the disease (spasticity, neuropathic pain, and fatigue), but by their nature they do not alter the course of the disease and there is a limited evidence base for symptomatic drug treatment for symptom control (Thompson 2001).

\section{Disease-modifying agents}

Of the five disease-modifying therapies approved by the FDA for the treatment of multiple sclerosis, four are immunomodulators (three preparations of interferon beta and glatiramer acetate) and one is an immunosuppressant (mitoxantrone). The diseasemodifying agents that are indicated for the treatment of RRMS include the immunomodulatory agents interferon beta-1b for subcutaneous administration (Betaseron ${ }^{\circledR}$ ), two formulations of interferon beta-1a for either subcutaneous (Rebif ${ }^{\circledR}$ ) or intramuscular administration $\left(\right.$ Avonex $\left.^{\circledR}\right)$, and glatiramer acetate $\left(\right.$ Copaxone ${ }^{\circledR}$ ). All four immunomodulators can be considered as first-line treatments for RRMS (Goodin et al. 2002; NMSS 2005).

Mitoxantrone (Novantrone ${ }^{\circledR}$ ) is an inhibitor of the enzyme DNA topoisomerase II which is responsible for uncoiling and repair of DNA in both dividing and nondividing cells. Because of concerns over cardiotoxicity this agent may only be used up to a cumulative lifetime dose of $\geq 140 \mathrm{mg} / \mathrm{m}^{2}$ (equivalent to about 11 doses) (Anon. 2005b). It is administered intravenously and due to toxic adverse effects it is generally reserved for the more progressive forms of the disease. Thus it is indicated for the treatment of worsening RRMS, SPMS, and PRMS. Recently, marketing of natalizumab (a humanized alfa-4 integrin antagonist) has been suspended because of reports of two serious adverse events (two cases of progressive multifocal leukoencephalopathy, one proving fatal). This agent had previously received accelerated approval in the USA in November 2004 for reducing the frequency of exacerbations in patients with RRMS after 1 year of treatment (FDA 2005).

The National Multiple Sclerosis Society (NMSS) has revised its consensus guidelines on the use of disease-modifying agents including interferon beta and glatiramer (NMSS 2005). The recommendations specify the use of the following four immunomodulators: interferon beta-1a (intramuscular), interferon beta-1a (subcutaneous), interferon beta-1b, and glatiramer acetate for all relapsing forms of multiple sclerosis and consideration of their use for selected patients with a first attack or who are at high risk of multiple sclerosis. Therefore, therapy is appropriate in all relapsing patients, those with SPMS, PPRS, and many patients experiencing a first attack, providing that no contraindication exists.

All of the agents approved for the treatment of RRMS have been shown to reduce relapse rates in large-scale, randomized, doubleblind, placebo-controlled, prospective trials (reviewed in Goodin et al. 2002). Both interferon beta-1a formulations have achieved reductions in sustained disability progression in relapsing multiple sclerosis when used during the early phase of the disease. For example, positive results have been obtained from a number of

\section{Table 2 | Disease course characteristics associated with the prognosis of multiple sclerosis}

Poor prognosis

Motor involvement, in particular disturbed coordination or balance

Onset of disease in older males

Frequent and prolonged relapses with incomplete recovery within 2 years of disease onset

Short interval between the initial episode and first relapse

Onset of progressive phase
Good prognosis

Sensory or visual symptoms dominate

Complete recovery from individual attacks 
separate 2-year placebo-controlled clinical trials involving patients with RRMS treated with the three interferon beta agents. In summary, interferon-beta treatment significantly reduced the relapse rate by 30 to $37 \%$ compared with placebo treatment (interferon beta-treated patient relapse rates ranged from 0.61 to 0.78 and placebo-treated rates from 0.9 to 1.2 relapses per year) (Compston \& Coles 2002). This change in relapse rate was also associated with a reduction in the accumulation of disability with the two interferon beta-1a (but not the interferon beta-1b) preparations.

In RRMS glatiramer acetate, mitoxantrone, and azathioprine all reduce relapse frequency and the accumulation of disability. Glatiramer acetate is a random polypeptide composed of four L-amino acids (glutamic acid, lysine, alanine, and tyrosine). Results from a placebo-controlled study involving 251 patients with RRMS showed that treatment with glatiramer acetate significantly reduced the clinical attack rate over a 2-year period by $27 \%$ ( $P=0.007$ vs placebo) (reviewed in Goodin et al. 2002). The indications for the use of glatiramer acetate are comparable to those for interferon beta and it is appropriate to consider it for treatment in any patient with RRMS (Goodin et al. 2002). For those patients who fail to adequately respond to the diseasemodifying agents, the only therapeutic option is to consider intensive immunosuppression with cytostatic agents or even autologous stem cell transplantation (Kappos et al. 2004).

Disease-modifying agents that can be started and continued on a long-term basis are referred to as "platform therapies." Key characteristics of an ideal agent used for platform therapy are maximal efficacy, safety, tolerability, convenience, and low rates of neutralizing antibody formation (neutralizing antibodies formed in the body may block or neutralize the biologic effects of the foreign protein or polypeptide, potentially decreasing the therapeutic effects of these agents) (Stuart et al. 2004). During periods of increased disease activity or instability other treatments (e.g. corticosteroids and immunosuppressants) may be used with platform therapy. Although almost all patients who recover from relapses do so spontaneously to some degree, most clinicians recommend treating a relapse if it has a significant effect on function (Polman \& Uitdehaag 2000). Corticosteroids have been the first-choice agent for this role for a number of years and although they shorten the duration of relapse and hasten recovery it is unclear whether they affect the overall degree of recovery or alter the course of the disease.

In summary, disease-modifying agents have beneficial effects on relapse rates, relapse-related disability, and MRI outcomes. These effects are more pronounced early in the course of the disease, are long lasting, and have no rebound effects (Kappos et al. 2004). Nevertheless these treatments are only partially effective; they are administered parenterally and although they are generally well tolerated there are some safety issues to be aware of (e.g. potential cardiotoxicity with mitoxantrone).

\section{Unmet needs}

One of the most important objectives of successful therapy for multiple sclerosis is the prevention or postponement of long- term disability. Typically, disability may evolve slowly over many years; however, most clinical trials are conducted for relatively short periods and only short-term outcome measures (e.g. attack rates and MRI measures to establish that treatment at least reduces the biologic activity of multiple sclerosis) are used. Therefore, it is important that any short-term measure is validated based on actual long-term patient outcomes (e.g. reduction in disability). Indeed, there is some uncertainty as to the relationship between the attack rate and long-term disability. It has been suggested that reducing short-term attack rate measures may not be associated with a delay in the accrual of disability in multiple sclerosis (reviewed in Goodin et al. 2002).

Based on results from a number of large, well-designed clinical trials it is generally accepted that interferon beta (1b or 1a) is the treatment of choice for patients with RRMS (Polman \& Uitdehaag 2000; Stuart 2004; Stuart et al. 2004). Nevertheless, there are still some unresolved issues relating to its use including optimal timing for the initiation and cessation of treatment; optimal dose, frequency, and route of administration; long-term effects of treatment; occurrence and relevance of neutralizing antibodies; and cost (Polman \& Uitdehaag 2000). In addition, up to $60 \%$ of patients experience influenza-like symptoms (including fever, chills, myalgia, and headache) with interferon beta (Calabresi 2004). The first-line choice for the treatment of SPMS is interferon beta; mitoxantrone or cyclophosphamide may be considered as second-line treatments for progressive disease. There are no established therapies for either PPMS or PRMS (Kieseier \& Hartung 2003).

Multiple sclerosis has a profound effect on patients' quality of life and it is important to determine the effect of any treatment on this parameter. At present, no study has measured this as a specific outcome of treatment. Instead, because the disease has been shown to be modified by treatment (e.g. reduced relapse rates and improvements in disability) this has led to the inference that quality of life outcomes are likely to be improved by these agents (NMSS 2005). However, this issue may be addressed through the use of a suitably valid and reliable quality of life instrument [e.g. the Multiple Sclerosis Impact Scale (MSIS29)].

Nevertheless, the management of multiple sclerosis has greatly benefited from the development of new disease-modifying agents such as interferon beta and glatiramer acetate as prior to their introduction there were no effective therapies. But, despite their widespread availability, they are still only partially effective (in terms of reductions in relapse rates, relapse-related disability, and imaging outcomes) in the treatment of multiple sclerosis, and all the currently available disease-modifying agents must be administered parenterally either by self-administration or under medical supervision. In addition, there is no agent currently available that is able to stop the disease process. Therefore, characteristics of an ideal agent for the treatment of multiple sclerosis would include oral administration (for convenience), clinically significant effects on disease- and patient-oriented outcomes, limitation of the disease process and reduced disability, and good tolerability. 


\section{Drug review}

Much progress has been made on the immunopathogenesis of the disease and many new promising therapeutic agents are currently in development. In general, these agents can be broadly categorized into either nonselective (antigen-nonspecific) or selective (antigen-specific) therapies (Hohlfeld \& Wekerle 2004). FTY720 (Fig. 2) is a new nonselective therapeutic agent in development for treatment of multiple sclerosis and will be reviewed in detail below. It is a novel, orally active compound derived from ISP-1 (myriocin), a fungal metabolite from Isaria sinclairii that was a remedy for "eternal youth" in traditional Chinese herbal medicine (Fujita et al. 1994). FTY720 is being developed by Novartis Pharma AG in the areas of transplantation and autoimmunity.

\section{Mode of action of FTY720}

FTY720 elicits lymphocyte sequestration by facilitating a reversible redistribution of lymphocytes from the circulation to secondary lymphoid tissues. This is a unique immunomodulation mechanism whereby $\mathrm{T}$ lymphocytes are effectively directed away from inflammatory sites toward the lymphatic system. Because of the structural similarity between FTY720 and sphingosine (a major component of the sphingolipids found in mammalian cell membranes; Fig. 2) it has been suggested that the drug may interact with sphingosine receptors (Brinkmann et al. 2002). Evidence suggests that FTY720 is phosphorylated in vivo via sphingosine kinase to give FTY720-phosphate (FTY720-P) which then participates in the sphingosine 1-phosphate (S1P) signaling cascade (Brinkmann \& Lynch 2002; Brinkmann et al. 2002).

S1P stimulates multiple cell signaling pathways by interacting with five (G-protein coupled) receptors, $\mathrm{S}_{1 \mathrm{P}_{1-5}}$. Distribution of these receptors shows that $\mathrm{S}_{1 \mathrm{P}_{1-3}}$ receptors are widely
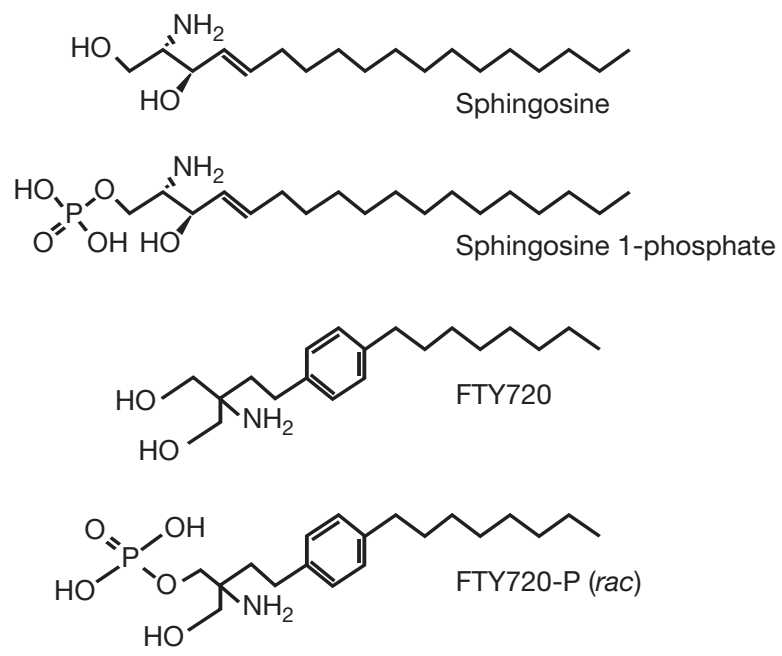

Fig. 2 | Structure of FTY720 and related compounds (adapted with permission from Brinkmann et al. 2002) expressed whereas the $\mathrm{S}_{1} \mathrm{P}_{4}$ receptor is specific to lymphoid tissue and $\mathrm{S}_{1} \mathrm{P}_{5}$ is found in the spleen and white matter tracts of the central nervous system (Brinkmann \& Lynch 2002). FTY720-P interacts as a high-affinity agonist at four of the five S1P receptors $\left(\mathrm{S}_{1} \mathrm{P}_{1}\right.$ and $\left.\mathrm{S}_{1} \mathrm{P}_{3-5}\right)$ (Brinkmann et al. 2002).

\section{Evidence of activity in animal models of multiple sclerosis}

Orally administered FTY720 is effective in a number of preclinical models of transplant rejection and autoimmune disease. The experimental autoimmune encephalomyelitis (EAE) model is one of the most widely used animal models of multiple sclerosis mimicking a number of pathologic characteristics of the disease. In a rat model, orally administered FTY720 $0.3 \mathrm{mg} / \mathrm{kg}$ per day prevented the development of EAE, as assessed by clinical disease score (Brinkmann et al. 2002). In another study using a rat model of EAE, oral FTY720 (0.3-1 mg/kg per day) completely eradicated inflammatory lesions in the central nervous system, as detected by either histology or MRI (Fujino et al. 2003; Rausch et al. 2004). Furthermore, when compared with control-treated animals in this study, FTY720 protected against both neurologic impairment and inflammatory lesions during the acute phase of the disease and subsequent first relapse (Rausch et al. 2004). These encouraging results suggest that FTY720 may be a promising candidate for clinical studies in the treatment of multiple sclerosis.

\section{Outcomes achieved with FTY720}

Pharmacokinetic and pharmacodynamic outcomes following single- or multiple-dose administration of FTY720 have been determined in both healthy subjects and transplantation patients (Table 3). These data are included here as these outcomes are not affected by disease status and may be extrapolated to include those patients with multiple sclerosis.

\section{Pharmacodynamic outcomes}

Results from a number of clinical studies have shown that FTY720 produces profound and reversible immunomodulation following oral administration. The mechanism of action of FTY720 leads to a reversible redistribution of lymphocytes from the circulation to secondary lymphatic tissue. The resulting lymphocyte sequestration is a convenient surrogate marker of the pharmacodynamic effect of FTY720 and may be a useful parameter for monitoring the immunomodulatory effect of the drug in the clinic.

There is substantial evidence that lymphocyte sequestration develops in healthy volunteers and renal transplant patients treated with FTY720 (Table 3). Administration of a single oral dose of FTY720 $1 \mathrm{mg}$ to 14 healthy volunteers resulted in a $38 \%$ reduction in the number of peripheral blood lymphocytes 2 days postdose (Kovarik et al. 2004b). In another study, the same dose led to a $44 \%$ reduction in the number of blood lymphocytes in 32 subjects with or without hepatic impairment (Kovarik et al. 2005). A similar effect was also seen in a phase I study after single-dose administration of FTY720 (0.25-3.5 mg) to 20 stable renal 
Table 3 | Summary of pharmacodynamic and pharmacokinetic outcomes for FTY720

\begin{tabular}{|ll}
\hline $\begin{array}{l}\text { Level of } \\
\text { evidence }\end{array}$ & Outcomes \\
\hline 2 & Drug exposure on d 7 was dose proportional for $C_{\max }(5.0 \pm 1.0$ \\
& vs $18.2 \pm 4.1 \mathrm{ng} / \mathrm{mL})$ and for AUC $(109 \pm 24$ vs $399 \pm 85 \mathrm{ng} / \mathrm{h}$ \\
& per $\mathrm{mL})$ for $\mathrm{FTY} 7201.25$ and $5 \mathrm{mg} / \mathrm{d}$, respectively \\
& Peripheral blood lymphocyte counts decreased from baseline to \\
& nadir (range $3-7 \mathrm{~d}$ after first dose) by 80 and by $88 \%$ in subjects \\
& receiving FTY720 1.25 and $5 \mathrm{mg}$, respectively. By $\mathrm{d} 35$ lymphocyte \\
& counts recovered to within 75 and $50 \%$ of baseline values in the \\
& FTY 1.25 and $5 \mathrm{mg}$ groups, respectively
\end{tabular}

Both $C_{\max }(0.65 \pm 0.17$ vs $0.64 \pm 0.18 \mathrm{ng} / \mathrm{mL})$ and AUC $(149 \pm 65$ vs $139 \pm 43 \mathrm{ng} / \mathrm{h}$ per $\mathrm{mL}$ ) were unchanged by the fasting or fed states, respectively

With both treatments the peripheral blood lymphocyte count decreased from baseline by $38 \pm 9 \%$ at $2 \mathrm{~d}$ postdose then increased towards predose values during the subsequent week

The absorption phase of FTY720 is prolonged, characterized by a $t_{\max }$ of $>12 \mathrm{~h}$

$C_{\max }\left(\mathrm{R}^{2}=0.966 ; P<0.001\right)$ and $\mathrm{AUC}\left(\mathrm{R}^{2}=0.916 ; P<0.001\right)$ were dose-proportional over the dose ranges $(0.25-3.5 \mathrm{mg})$

All FTY720 groups showed a temporal pattern of relative lymphocyte sequestration, seen at the latest $6 \mathrm{~h}$ postdose. No clear dose response, but the highest doses showed a more pronounced reduction in lymphocyte numbers. Lymphocyte counts returned to $\sim 80 \%$ baseline values $24 \mathrm{~h}$ postdose

Almost all lymphocyte subgroups declined following FTY720 treatment, with CD4+ and CD45RA+ cells being affected the most. Natural killer cells, granulocytes, and monocytes were not influenced by FTY720

Steady-state concentrations of FTY720 were achieved by w 4 . In the 10-fold dose range studied the pharmacokinetic profile of FTY720 was linear with dose $\left(R^{2}=0.679\right.$ and 0.982 for individual and mean FTY720 concentration, respectively)

CS doses and whole blood concentrations were unaffected by FTY720 (or MMF). Therapeutic concentrations of CS were achieved in all groups during the study period, and required no dose adjustment

During 12-w treatment with FTY720 lymphocyte sequestration was seen as early as $w 1$, nadir was reached at w 4 and was fully reversed 4-8 $w$ after cessation of treatment. The pharmacodynamics were not dose-linear over the 10 -fold dose range $\left(R^{2}=0.57\right.$ and 0.53 for lymphocyte sequestration vs drug dose and vs drug concentrations, respectively). Lymphocyte sequestration was not seen in the MMF-treated group

$C_{\text {max }}$ was not influenced by hepatic function $(0.64 \pm 0.17$, $0.65 \pm 0.12,0.57 \pm 0.10$ for controls, mild impairment, and

$0.25(n=4), 0.5(n=4)$, $1(n=5)$, or $2.5 \mathrm{mg}(\mathrm{n}=5)$, vs MMF $2 \mathrm{~g}(\mathrm{n}=5)$

$(n=6), 0.5(n=6)$

$.75(n=3), 1(n=3)$

$2(\mathrm{n}=3)$, or $3.5 \mathrm{mg}$

$(n=3)$, or placebo $(n=8)$

mg under fasting conditions or with crossover, single-dose study in 14 healthy male volunteers (age range 20-39 y)

Randomized, double-blind, placebo-controlled, two-center, single-dose study in 20 stable renal transplant patients (mean age $43.2 \mathrm{y}$ )
Reference

Kovarik et al. 2004a

placebo-controlled,

multiple-dose study

in 60 healthy volunteers

(age range 18-44 y)

Kovarik et al. 2004b

Budde et al. 2002;

Budde et al. 2003

Randomized, multiple-dose,

Park et al. 2005 treatment then $12-w$ follow-up). 23 renal transplant recipients (mean age $40.1 \mathrm{y}$ ). All patients were treated with CS and PD moderate impairment, respectively). Oral clearance was reduced on average by 10 and $31 \%$ for mild and moderate hepatically impaired subjects compared with the respective controls, reduced metabolism being the likely cause

The magnitude of the pharmacokinetic changes suggests that the FTY720 dose need not be adjusted in patients with mild or moderate hepatic impairment

The effect on lymphocyte sequestration was similar across all groups with a mean decrease of $44 \%$ from the predose baseline

AUC, area under the concentration-time curve; $C_{\max }$, peak plasma concentration; CS, cyclosporine; d, day; h, hour; $\mathrm{R}^{2}$, correlation coefficient; $t_{\max }$, time to $C_{\max } ;$ MMF, mycophenolate mofetil; $\mathrm{PD}$, prednisone; $w$, week; $y$, year.

transplant patients receiving a cyclosporine-based regimen (Budde et al. 2002). Although the higher doses of FTY720 produced a more rapid and sustained lymphocyte sequestration, the actual degree of this property was similar across the range of doses used in the study and no clear dose-response relationship was detected. An analysis of the subsets of lymphocytes sensitive to FTY720 was also performed in this study. The effect of FTY720 was seen across all lymphocyte subsets except for natural killer cells. As early as $4 \mathrm{~h}$ postdose, profound effects on the different lymphocytes subsets were seen, with CD4+ and naïve T-lymphocyte counts decreasing to the greatest extent (Budde et al. 2003). 
The profound lymphocyte sequestration observed with multiple doses of FTY720 has also been shown to be reversible after cessation of drug treatment. For example, in a placebo-controlled study lymphocyte counts decreased by 80 and $88 \%$ in healthy subjects receiving orally administered FTY720 1.25 or 5 mg/day, respectively (Kovarik et al. 2004a). The lymphocyte counts were reduced at the first postdose sampling time point on day 2 . During the washout phase (between days 9 and 35) the lymphocyte counts recovered towards baseline values. Similar outcomes were also seen in a study with patients treated with FTY720 0.25-2.5 mg/day for 12 weeks following renal transplantation (Park et al. 2005). FTY720-induced lymphocyte sequestration was observed during the first week of treatment and the nadir was reached by week 4 . This effect was fully reversed 4-8 weeks after stopping treatment.

\section{Pharmacokinetic outcomes}

The pharmacokinetic parameters of FTY720 are not influenced by disease status and therefore these outcomes that have been derived from studies in healthy volunteers and transplant recipients are valid for patients with multiple sclerosis.

Results from a number of phase I studies with FTY720 have shown that the pharmacokinetic parameters following daily oral administration are predictable and are not influenced by food intake or impaired hepatic function (Table 3). Therefore, oral administration of FTY720 is likely to be convenient and require minimal dose adjustment when used in the clinic.

There is substantial evidence to show that following oral administration the absorption phase of FTY720 is prolonged, characterized by a $t_{\max }$ of $>12 \mathrm{~h}$ (Budde et al. 2002; Kovarik et al. $2004 a, b, 2005)$. The pharmacokinetic parameters of FTY720 are similar in healthy subjects and transplant recipients (Kovarik et al. 2004a). Hence pharmacokinetic data derived from healthy subjects can be used to determine dosage guidelines for patients (Kovarik et al. 2004a,b). Studies in healthy subjects have indicated that FTY720 may be administered without regard to the timing of meals and that no dosage alteration is necessary in patients with mild or moderate hepatic impairment (Kovarik et al. 2004b, 2005).

\section{Clinical outcomes}

Thus far in its development for the treatment of multiple sclerosis, only meeting abstracts covering one study have been reported. This was a placebo-controlled study which showed that orally administered FTY720 achieved promising patientand disease-oriented outcomes compared with placebo treatment after 6 months, and continued with a subsequent 6-month extension.

In this phase II international multicenter trial, 281 patients with active relapsing multiple sclerosis were randomized to receive FTY720 $1.25 \mathrm{mg}(\mathrm{n}=94)$, or $5 \mathrm{mg}(\mathrm{n}=94)$, or placebo $(\mathrm{n}=93)$ once daily for 6 months (Table 4; Kappos et al. 2005). The primary outcome was inflammatory disease activity as assessed by the mean total number of gadolinium (Gd)-enhancing lesions in monthly postbaseline MRI scans. Compared with placebo, FTY720 1.25 and $5 \mathrm{mg} /$ day significantly reduced inflammatory disease activity by 43 and $61 \%$, respectively $(P \leq 0.006)$. Similarly, new disease activity (as measured by MRI) was also reduced by FTY720 (actual data not provided). In addition, when compared with placebo treatment the annual relapse rate was significantly reduced by 55 and $53 \%$ with FTY720 1.25 and $5 \mathrm{mg} /$ day, respectively ( $P$ values not provided in the abstract). A total of $86 \%$ of patients in both FTY720-treated groups were relapse-free compared with $70 \%$ of placebo-treated patients $(P \leq 0.008)$ after 6 months.

Table 4 | Summary of outcomes achieved with FTY720 in clinical trials of multiple sclerosis

\begin{tabular}{|c|c|c|c|c|}
\hline $\begin{array}{l}\text { Level of } \\
\text { evidence }\end{array}$ & Outcomes & FTY720 dosage & Study design and population & Reference \\
\hline $2^{a}$ & $\begin{array}{l}\text { Inflammatory disease activity (mean total number of Gd-enhancing } \\
\text { lesions in monthly postbaseline MRI scans) was significantly reduced } \\
\text { by } 43(P<0.001 \text { vs placebo) and } 61 \% \text { ( } P=0.006 \text { vs placebo) with } \\
\text { FTY720 } 1.25 \text { and } 5 \mathrm{mg} \text {, respectively. The annualized relapse rate was } \\
0.77 \text { (placebo), } 0.35 \text { (FTY720 } 1.25 \mathrm{mg} \text { ), and } 0.36 \text { (FTY720 } 5 \mathrm{mg} \text { ) a } \\
\text { reduction of } 55 \text { and } 53 \% \text { with FTY720 } 1.25 \text { and } 5 \mathrm{mg} \text {, respectively, } \\
\text { vs placebo } \\
86 \% \text { of FTY720-treated patients were relapse-free after } 6 \text { months } \\
\text { compared with } 70 \% \text { of placebo-treated patients ( } P=0.007 \text { for } \\
\text { FTY720 } 1.25 \mathrm{mg}, P=0.008 \text { for FTY720 } 5 \mathrm{mg})\end{array}$ & $\begin{array}{l}1.25(n=94) \text { and } 5 \mathrm{mg} \\
(n=94) \text { vs placebo }(n=93)\end{array}$ & $\begin{array}{l}\text { Randomized, multicenter, } \\
\text { double-blind, placebo-controlled } \\
281 \text { patients with active relapsing } \\
\text { multiple sclerosis }\end{array}$ & $\begin{array}{l}\text { Kappos et al. } \\
2005\end{array}$ \\
\hline $2^{a}$ & $\begin{array}{l}\text { Annualized relapse rates reduced by }>50 \% \text { following switch from } \\
\text { placebo to FTY720 } \\
\text { For patients continually treated with FTY720 } 1.25 \text { and } 5 \mathrm{mg} \text {, } \\
80 \text { and } 83.8 \% \text { were relapse-free, respectively } \\
84.3 \text { and } 89.1 \% \text { patients treated with FTY720 } 1.25 \text { and } 5 \mathrm{mg} \text { were } \\
\text { free from Gd-enhanced lesions at } 12 \text { months, respectively }\end{array}$ & $\begin{array}{l}1.25(n=87) \text { and } 5 \mathrm{mg} \\
(\mathrm{n}=80), \text { plus placebo to } \\
1.25 \mathrm{mg}(\mathrm{n}=40) \text {, and } \\
\text { placebo to } 5 \mathrm{mg}(\mathrm{n}=43)\end{array}$ & $\begin{array}{l}250 \text { patients in } 6 \text {-month extension } \\
\text { phase } \\
\text { Patients switched from placebo } \\
\text { to FTY720 after } 6 \text { months }\end{array}$ & $\begin{array}{l}\text { O'Connor } \\
\text { et al. } 2005\end{array}$ \\
\hline
\end{tabular}


In the extension phase, patients continued on their blinded FTY720 treatment while those assigned to placebo switched to either dose of FTY720 (O'Connor et al. 2005). Results showed that the annualized relapse rates were reduced by over $50 \%$ following switch from placebo to FTY720. After 12 months' treatment with FTY720 1.25 and 5.0 mg/day, 80.0 and $83.8 \%$ of patients, respectively, remained relapse-free. In addition, MRI assessments at 12 months showed that $84.3 \%$ in the $1.25 \mathrm{mg} /$ day group and $89.1 \%$ in the $5.0 \mathrm{mg} /$ day group were free from Gd-enhanced lesions (Table 4).

\section{Safety and tolerability outcomes}

There is good evidence that FTY720 is well tolerated in singleand multiple-dose studies (Table 5). No serious adverse events have been noted in any study. Reported adverse events that may be treatment related include headache, dizziness, and nausea. However, it is unclear from the reports as to what the incidences of these events are following treatment with FTY720.

Data from studies in healthy volunteers and transplant recipients have been included as they provide drug-specific outcomes that are relevant to all patients, irrespective of their disease status.

A commonly observed effect of FTY720 is a mild, asymptomatic, transient reduction in heart rate which is not clinically relevant. Supine heart rate shows a distinct circadian rhythm, which is preserved following treatment with FTY720 (Kovarik et al. 2004b). However, when compared with placebo, FTY720-associated reduction in heart rate can be demonstrated by a downward shift in the heart rate versus time curve by approximately 10\% (Kovarik et al. 2004b, 2005). This effect is consistent with agonist properties on S1P receptors in the sinus node and atrial cells of the heart and has been observed in preclinical models (Brinkmann \& Lynch 2002). The reduction in heart rate was reversible and required no clinical intervention, and the effect was shown not to be related to FTY720 blood concentrations (Budde et al. 2002). All patients were asymptomatic, blood pressure remained within normal limits, and no orthostatic reaction was seen in any patient.

There is good evidence from the clinical studies thus far suggesting that the lymphocyte sequestration seen following administration of FTY720 for up to 6 months is not associated with the development

Table 5 | Summary of outcomes associated with safety and tolerability achieved with FTY720 in clinical development

\begin{tabular}{|c|c|c|c|c|}
\hline $\begin{array}{l}\text { Level of } \\
\text { evidence }\end{array}$ & Outcomes & FTY720 dosage & Study design and population & Reference \\
\hline \multirow[t]{2}{*}{2} & $\begin{array}{l}\text { A total of } 114 \text { AEs reported by } 36 \text { subjects receiving FTY720 and } \\
\text { placebo; } 65 \text { and } 21 \% \text { of the AEs were reported during the treatment } \\
\text { and washout phases, respectively. About half of the AEs consisted } \\
\text { of headache }(31 \%) \text {, dizziness }(9 \%) \text {, and nausea }(7 \%)\end{array}$ & $\begin{array}{l}1.25(n=20) \text {, or } 5 \mathrm{mg} \\
(\mathrm{n}=20) \text {, or placebo } \\
(\mathrm{n}=20) \text { once daily for } \\
7 \mathrm{~d}\end{array}$ & $\begin{array}{l}\text { Randomized, double-blind, } \\
\text { placebo-controlled, multiple-dose } \\
\text { study in } 60 \text { healthy volunteers } \\
\text { (age range } 18-44 \text { y) }\end{array}$ & $\begin{array}{l}\text { Kovarik et al. } \\
2004 a\end{array}$ \\
\hline & AE distribution was 16,48 , and $36 \%$ for FTY720 1.25 mg, & & & \\
\hline
\end{tabular}
FTY720 5 mg, and placebo groups, respectively

FTY720 preserved supine heart rate circadian rhythm. The heart rate vs time curve was shifted downwards by $10 \%$ over the first day postdose, recovering to prestudy values after 3-5 d postdose. All changes were asymptomatic and unaffected by the fasting or fed states

No serious AEs seen in the study. AEs occurred in $91 \%$ of FTY720-treated subjects vs $75 \%$ of placebo-treated subjects. Of the 28 AEs reported the most common were transient asymptomatic reduction in heart rate $(n=10)$ and headache

$0.25(n=6), 0.5(n=6)$, $0.75(n=3), 1(n=3)$, $1 \mathrm{mg}$ under fasting conditions or with a high-fat meal
Randomized, two-period, crossover study in 14 healthy male volunteers (age range 20-39 y)
Kovarik et al. 2004b

\section{$2(n=3)$, or $3.5 \mathrm{mg} \quad$ study in 20 stable renal}

$(n=3)$, or placebo $(n=8)$ transplant patients (mean age $43.2 y$ )

$1.25(n=94)$ and $5 \mathrm{mg}$ Randomized, multicenter, $(\mathrm{n}=94)$ vs placebo $(n=93)$

Randomized, multicenter,
double-blind, placebo-controlled

Kappos et al. AEs were more frequently reported in the $5 \mathrm{mg}$ group and the and nasopharyngitis

281 patients with active relapsing multiple sclerosis

the study and $89 \%$ elected to continue to the extension phase

15 AEs reported in 9 subjects; 12 AEs in 7 hepatic-impaired postdose and recovering within 1-2 d

${ }^{\mathrm{a} A b s t r a c t .}$

$\mathrm{AE}$, adverse event; $d$, day; h, hour; y, year. 
of adverse effects. For example, no increased risk of infections has been seen in renal transplant patients (Budde et al. 2002).

The adverse events noted most frequently in the 12-month clinical study in patients with relapsing multiple sclerosis were limited to nasopharyngitis, influenza, and headache reported by $>15 \%$ of patients in at least one group treated with FTY720 (1.25 or 5 mg/day) (O'Connor et al. 2005).

\section{Resource utilization}

Because FTY720 is still in the early stages of clinical development no data has yet appeared indicating its value in resource utilization. However, there are outcomes which may be measured in future studies that will provide evidence for its utility in this area. Such outcomes may include cost savings resulting from reduction in long-term disability or reductions in the management of adverse events. Reductions in relapse rates and frequency and duration of attacks will also influence potential savings.

The management of multiple sclerosis has benefited from the development and availability of newer disease-modifying agents. Nevertheless, although these newer therapies are able to improve both patient-oriented (e.g. relapse rates and disability) and disease-oriented (e.g. MRI-based data) outcomes their effects are relatively modest. These treatments are also lifelong therapies. Given that a patient may have the disease for several decades the acquisition cost for these drugs alone will be substantial. There will also be instances where the disease-modifying agents fail to be effective in some patients. In these cases the therapeutic options that may be considered include cytostatic agents to achieve intensive immunosuppression and autologous stem cell transplantation (Kappos et al. 2004). These options will also have cost implications including costs associated with the management of adverse events associated with immunosuppression and processes surrounding transplantation.

All the current disease-modifying agents are administered by injection, there being no conveniently administered oral agent currently available. Mitoxantrone is administered intravenously under medical supervision every 3 months and intramuscular interferon beta-1a (Avonex) also requires medical guidance and supervision for administration. Interferon beta-1a (Rebif), interferon beta-1b (Betaseron), and glatiramer acetate may all be self-administered subcutaneously but this requires medical guidance initially. An effective orally administered preparation on the other hand is likely to be more convenient for the patient and reduce medical involvement.

One of the most debilitating effects of multiple sclerosis is long-term disability. This may impact significantly on patient quality of life over many years and frequently requires significant resources to support patients, their family, and/or dependents. The Expanded Disability Status Scale (EDSS) which ranges from 1 (least severe) to 10 (death from multiple sclerosis) is a commonly used instrument for grading the functional effects of multiple sclerosis and is the only standardized testing element common to both clinical trials and natural history studies. Cost studies in the UK have shown that, on average, patients with less severe disease (EDSS $1-3.5)$ incur annual costs of around $£ 3350$ compared with $£ 9560$ for those patients with more severe disease (EDSS 6.5-8.) (Richards et al. 2002). Therefore any treatment that can effectively reduce the disability status of patients is likely to have a considerable impact on quality of life and the economic burden of the disease.

\section{Patient group/population}

Thus far FTY720 has been evaluated in a 12-month trial in patients with active RRMS and it is likely that further studies will be conducted in patients with this form of the disease. One reason for this is that RRMS is the most prevalent form of the disease. Nevertheless it should be noted that as yet there are no effective therapies for the least common types of multiple sclerosis, PPMS, or PRMS.

\section{Drug profile}

Progress has been achieved in the treatment of multiple sclerosis with the availability of newer disease-modifying agents. In particular, these agents have shown some success in treating the most common form of the disease, RRMS. However, there are a number of limitations associated with the use of interferon beta, glatiramer acetate, and mitoxantrone for treating multiple sclerosis. For example, many patients treated with interferon beta experience influenza-like symptoms, and although these may be managed by pretreatment with nonsteroidal antiinflammatory drugs, some patients may discontinue treatment. Also potential cardiotoxicity associated with mitoxantrone restricts its use to the treatment of the more aggressive forms of the disease without exceeding the lifetime cumulative dose limit. Although diseasemodifying agents have shown efficacy, their impact on the course of the disease is relatively modest. In addition, their acquisition cost is high and parenteral administration can be inconvenient. Less costly oral immunomodulatory agents are available (e.g. azathioprine, cyclophosphamide), but they have less favorable adverse-event profiles. In addition, there is no established effective treatment for either PPMS or PRMS. Therefore, there is the need for new, effective, and well-tolerated treatments, particularly for oral administration, for multiple sclerosis.

The novel S1P receptor agonist FTY720 is undergoing clinical development for use in transplantation and autoimmunity. There is substantial published evidence showing that FTY720 can achieve reliable and reversible immunomodulation (lymphocyte sequestration) following oral administration. In addition, there is good evidence that the pharmacodynamic and pharmacokinetic profile of the drug is unaffected by the timing of meals or hepatic impairment.

FTY720 has shown promising results in preclinical models of EAE, which in part has led to its clinical evaluation in multiple sclerosis. There is moderate evidence from two meeting abstracts of a phase II study that FTY720 (administered orally once daily for up to 12 months) improved the patient-oriented outcomes of relapse 
rate and the likelihood of remaining relapse-free. In addition, there is moderate evidence that disease-oriented outcomes were also improved by FTY720 in that inflammatory disease activity (both new and existing) was reduced as determined by MRI.

Based on these early results of efficacy and tolerability from the clinical development program, FTY720 has the potential to be an effective disease-modifying agent for the treatment of RRMS. It has the advantage over currently available treatments that it is orally administered and has also exhibited a favorable tolerability profile from the studies so far conducted. Further results from ongoing multinational phase III studies are awaited. In particular, data on its long-term effects on outcomes in the treatment of RRMS (e.g. reductions in disability), utility for other forms of multiple sclerosis, and tolerability will be anticipated.

\section{References}

Anon. Multiple sclerosis - FTY720, a novel once-daily oral medication, shows promising results. Medical News Today. June 25, 2005. Available at: http://www.medicalnewstoday.com/medicalnews.php?newsid=26611 (accessed July 5, 2005a).

Anon. Novantrone Prescribing Information. Available at: http://www.novantrone.com/assets/pdf/novantrone_prescribing_info.pdf (accessed August 1, 2005b).

Brinkmann V, Lynch KR. FTY720: targeting G-protein-coupled receptors for sphingosine 1-phosphate in transplantation and autoimmunity. Curr Opin Immunol. 2002;14:569-575.

Brinkmann V, Davis MD, Heise CE, et al. The immune modulator FTY720 targets sphingosine 1-phosphate receptors. J Biol Chem. 2002;277:21453-21457.

Budde K, Schmouder RL, Brunkhorst R, et al. First human trial of FTY720, a novel immunomodulator, in stable renal transplant patients. J Am Soc Nephrol. 2002;13:1073-1083.

Budde K, Schmouder RL, Nashan B, et al. Pharmacodynamics of single doses of the novel immunosuppressant FTY720 in stable renal transplant patients. Am J Transplant. 2003;3:846-854.

Calabresi PA. Diagnosis and management of multiple sclerosis. Am Fam Physician. 2004;70:1935-1944

Compston A, Coles A. Multiple sclerosis. Lancet. 2002;359:1221-1231.

Dyment DA, Sadnovich AD, Ebers GC. Genetics of multiple sclerosis. $\underline{\text { Hum Mol }}$ Genet. 1997;6:1693-1698.

FDA (Food and Drug Administration). Public Health Advisory. Suspended marketing of Tysabri (natalizumab). Available at:

http://www.fda.gov/cder/drug/advisory/natalizumab.htm (accessed June 9, 2005).

Fujino M, Funeshima N, Kitazawa Y, et al. Amelioration of experimental autoimmune encephalomyelitis in Lewis rats by FTY720 treatment. J Pharmacol Exp Ther. 2003;305:70-77.

Fujita T, Inoue K, Yamamoto S, et al. Fungal metabolites. Part 11. A potent immunosuppressive activity found in Isaria sinclairii metabolite. $J$ Antibiot (Tokyo). 1994;47:208-215.

Goodin DS, Frohman EM, Garmany GP Jr, et al. Disease modifying therapies in multiple sclerosis: report of the Therapeutics and Technology Assessment Subcommittee of the American Academy of Neurology and the MS Council for Clinical Practice Guidelines. Neurology. 2002;58:169-178 [including supplemental data available at: http:///www.neurology.org/cgi/data/58/2/169/DC3/1 (accessed August 9, 2005)].

Hafler DA. The distinction blurs between an autoimmune versus microbial hypothesis in multiple sclerosis. J Clin Invest. 1999;104:527-529.

Hafler DA. Multiple sclerosis. J Clin Invest. 2004;113:788-794.

Hohlfeld R, Wekerle H. Autoimmune concepts of multiple sclerosis as a basis for selective immunotherapy: from pipe dreams to (therapeutic) pipelines. Proc Natl Acad Sci U S A. 2004;101(Suppl. 2):14599-14606.
Kappos L, Kuhle J, Gass A, et al. Alternatives to current disease-modifying treatment in MS: what do we need and what can we expect in the future? J Neurol. 2004;251(Suppl. 5):v57-v64.

Kappos L, Radue EW, Antel J, et al. FTY720 in relapsing MS: results of a double-blind placebo-controlled trial with a novel oral immunomodulator. J Neurol. 2005;252(Suppl. 2) (Abstract O140).

Kieseier BC, Hartung H-P. Current disease-modifying therapies in multiple sclerosis. Semin Neurol. 2003;23:133-146.

Kovarik JM, Schmouder R, Barilla D, Riviere GJ, Wang Y, Hunt T. Multiple-dose FTY720: tolerability, pharmacokinetics, food and lymphocyte responses in healthy subjects. J Clin Pharmacol. 2004a;44:532-537.

Kovarik JM, Schmouder R, Barilla D, Wang Y, Kraus G. Single-dose FTY720 pharmacokinetics, food effect, and pharmacological responses in healthy subjects. Br J Clin Pharmacol. 2004b;57:586-591.

Kovarik JM, Schmouder RL, Serra D, et al. FTY720 pharmacokinetics in mild to moderate hepatic impairment. J Clin Pharmacol. 2005;45:446-452.

Lutton JD, Winston R, Rodman TC. Multiple sclerosis: etiological mechanisms and future directions. Exp Biol Med. 2004;229:12-20.

Miller DH, Grossman RI, Reingold SC, McFarland HF. The role of magnetic resonance techniques in understanding and managing multiple sclerosis. Brain. 1998;121:3-24.

Mohr DC, Hart SL, Julian L, Cox D, Pelletier D. Association between stressful life events and exacerbation in multiple sclerosis: a meta-analysis. BMJ. 2004;328:731.

NMSS (National Multiple Sclerosis Society). Disease management consensus statement. Available at: http://www.nationalmssociety.org/pdf/forpros/ exp_consensus.pdf (accessed July 12, 2005).

Noseworthy JH, Lucchinetti C, Rodriguez M, Weinshenker BG. Multiple sclerosis. N Engl J Med. 2000;343:938-952.

O'Connor P, Antel J, Comi X, et al. Phase II study with oral FTY720 in relapsing MS: results of the dose-blinded active drug extension phase at 12 months. Mult Scler. 2005;11(Suppl. 1) (Abstract 682).

Park SI, Felipe CR, Machado PG, et al. Pharmacokinetic/pharmacodynamic relationships of FTY720 in kidney transplant recipients. Braz J Med Biol Res. 2005;38:683-694.

Polman $\mathrm{CH}$, Uitdehaag BMJ. Drug treatment of multiple sclerosis. BMJ. 2000;32:490-494.

Rausch M, Hiestand P, Foster CA, Baumann DR, Cannet C, Rudin M. Predictability of FTY720 efficacy in experimental autoimmune encephalomyelitis by in vivo macrophage tracking: clinical implications for ultrasmall superparamagnetic iron oxide-enhanced magnetic resonance imaging. J Magn Res Imaging. 2004;20:16-24.

Richards RG, Sampson FC, Beard SM, Tappenden P. A review of the natural history and epidemiology of multiple sclerosis: implications for resource allocation and health economic models. Health Technol Assess. 2002;6:1-73.

Stuart WH. Clinical management of multiple sclerosis: the treatment paradigm and issues of patient management. J Manag Care Pharm.

2004;10(Suppl. B):S19-S25.

Stuart WH, Cohan S, Richert JR, Achiron A. Selecting a disease-modifying agent as platform therapy in the long-term management of multiple sclerosis. Neurology. 2004;63(Suppl. 5):S19-S27.

Thompson AJ. Symptomatic management and rehabilitation in multiple sclerosis. J Neurol Neurosurg Psychiatry. 2001;71(Suppl. II):ii22-ii27.

Weinshenker BG. Natural history of multiple sclerosis. Ann Neurol. 1994;36(Suppl.):S6-S11.

Correspondence: Andrew Thomson, Core Medical Publishing, Mere House, Brook Street, Knutsford, Cheshire WA16 8GP, UK or at editor@coreevidence.com 